\title{
Calculating cargo securing elements on a railway platform under the impact of a spatial force system
}

\author{
Khabibulla Turanov ${ }^{1}$, Yadgor Ruzmetov ${ }^{2, *}$, and Natalia Vlasova ${ }^{3}$ \\ ${ }^{1}$ Ural State University of Railway Transport, Kolmogorova st., 66, 620034, Yekaterinburg, Russia \\ ${ }^{2}$ Tashkent Railway Engineering Institute, Temirylchilar 1- tor kycha, 1, 100167, Tashkent, \\ Uzbekistan \\ ${ }^{3}$ Irkutsk State Transport University, Chernyshevskogo str., 15, Irkutsk, Russia
}

\begin{abstract}
Form a design (or dynamic) model on the basis of studying the physical model of a mechanical system (object); build mathematical models of cargo securing with inclined side surfaces of stop elements (bars) with simultaneous influence of longitudinal and lateral forces; perform a mathematical solution of the problem in the form of specific analytical formulas for calculating inclined stop bars as one of the cargo securing elements on the railway platform; make a specific example of the calculation of the forces acting on the cargo securing elements.
\end{abstract}

\section{Introduction}

Analysis of scientific works, for example, [1 - 8], makes it possible to note that a dynamic and mathematical model of securing cargo with inclined side surfaces with stop elements (bars) with simultaneous impact of longitudinal and lateral forces (hereinafter - spatial force systems) has not yet been built. Note that a characteristic defect in the transportation of machinery, equipment and reinforced concrete products is the stretching and tearing of the guy ropes and, as a rule, the longitudinal shear of both the entire cargo and the individual cargo units. The current technical specifications (TS) for the carriage of goods in a car establish the ways of placing and securing goods that meet certain requirements. However, cargo of various configurations that are not provided by the technical specifications is presented for carriage. Therefore, the sender must calculate the fastening for each such cargo. For example, the results of the study [9-12] showed that they mainly describe the definition of forces in elastic and stop fasteners of the cargo when subjected to separate longitudinal and separate lateral forces. In this case, the forces in all elastic fasteners, regardless of their location (i.e. at an angle or perpendicular to the side surfaces of the cargo) relative to the side surfaces of the cargo, have the same values, which is erroneous. As a result, this leads to a breakdown in the placement and fastening in transit, and is the cause of the unsafe transportation of goods, which can lead to a safety threat of train traffic.

* Corresponding author: turanov@yandex.ru 
The acceleration of the car along the longitudinal and lateral axis of the car is mainly studied. The standards for calculating the cargo securing in fractions g are established $(4 \mathrm{~g}$ in the longitudinal direction, $0.4 \mathrm{~g}$ in the lateral direction, $0.3 \mathrm{~g}$ in the vertical direction). They recorded that the coefficient of friction between the contacting surfaces decreases by almost 30\%, which is the cause of the longitudinal and lateral displacements of the cargo in the car. At the same time, in [4], there are no calculations for determining the forces in the cargo fasteners. The results of both analytical and calculated data on the determination of forces in elastic fasteners, depending on their location relative to the side surfaces of the cargo, are stated in [12], where it is proved that the forces in elastic fasteners depend on their fastening geometry and have different values, which corresponds to reality.

As can be seen, in [1-12], the problems of calculating inclined stop elements for fastening (bars) cargo to the floor of a car under the simultaneous impact of spatial force systems are not studied at all.

The objective of this study:

- formulate the conditions of the problem and the accepted assumptions in the preparation of a dynamic model. It should be borne in mind that in the dynamic model, the cargo securing elements are affected by spatial systems of forces that are perceived by the main constraint (car) and additional constraints (elastic and stop wooden fasteners);

- form a design (or dynamic) model of securing cargo in the car on the basis of the study of physical model of the mechanical system (object);

- build mathematical models of cargo securing with inclined side surfaces by means of stop elements (bars) under the influence of spatial force systems;

- perform a mathematical solution of the problem in the form of specific analytical formulas for calculating inclined stop bars, as one of the cargo securing elements on the railway platform;

- make a specific example of the calculation of the forces acting on the stop elements for securing cargo.

\section{Research methods}

The research methods are based on the most important principles of classical mechanics (for example, the concepts "constraints" and "constraint reaction", the principle of replacing constraints of geometric statics, Coulomb's law, Hooke's law, equilibrium conditions of a rigid body during relative motion (rest), and the notion of "shearing" and "retentive" forces). For example, to build a dynamic cargo model (object) with cargo loops in the presence of stop bars, according to the principle of replacing geometric constraints, external constraints (the platform frame as the main constraint, and elastic securing elements as additional constraints) are mentally rejected from an object.

\section{Research results}

Formulation of the conditions of the problem and the accepted assumptions in the preparation of a dynamic model. Let us consider the case where the cargo of gravity force $\mathrm{G}$, which is symmetrically placed relative to the longitudinal and lateral axis of symmetry of the car. One of the examples of the scheme (or drawing) of placing and securing cargo in the practice of cargo transportation is shown in Fig. 1 in the form of a physical model. 


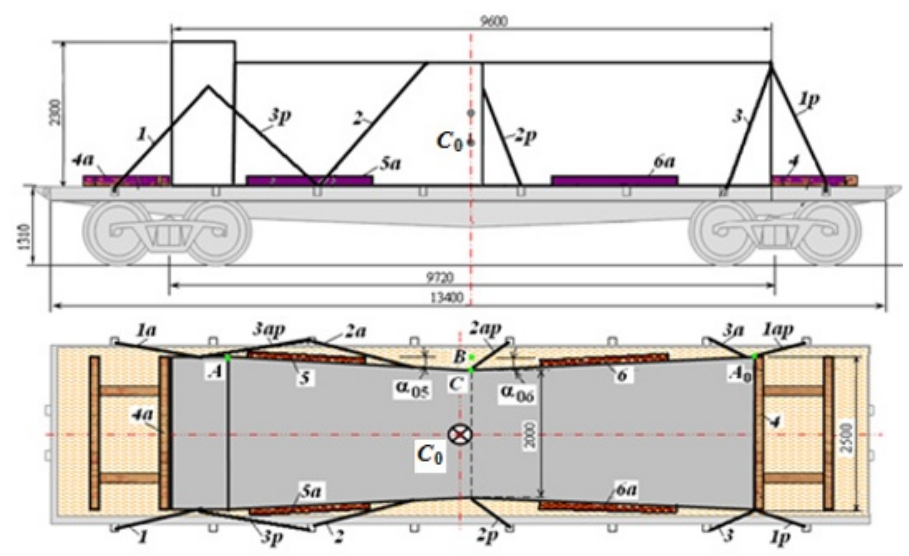

Fig. 1. The scheme of placing and securing cargo on a car with elastic and stop elements. $1-3,1 p-$ $3 p, 1 a-3 a, 1 a p-3 a p$ - elastic elements of fastenings of one and other direction; $4-6,4 a-6 a-$ stop bars; $\alpha_{05}$ and $\alpha_{06}-$ angles of inclination of the side surfaces of the stop bars 5 and 6 .

The elastic elements of the fasteners and the frame of the car as external constraints experience simultaneous effects of longitudinal, vertical and lateral forces - $\bar{G}, \bar{I}_{e}$ $\left(\bar{I}_{e}=\left\{\bar{I}_{e x}, \bar{I}_{e y}, \bar{I}_{e z}\right\}\right.$ and $\bar{F}_{r \mathrm{~B}}\left(\bar{F}_{r \mathrm{~B}}=\left\{\bar{F}_{r \mathrm{~B} x}^{\prime}, \bar{F}_{r \mathrm{~B} y}^{\prime}\right\}\right)$ - the gravity force of the car with cargo (or without cargo), portable inertia forces [15] and the force of aerodynamic resistance. We believe that the longitudinal, lateral and vertical forces are perceived by elastic fastening elements that are opposite to the action of these forces, and fastening elements of the opposite direction are sagging (i.e. losing the properties of constraints). We assume that the cargo is massive and does not detach from the floor of the car, and the cargo does not overturn with respect to its side and end faces, i.e. the cargo will not lose constraint in the form of the car's floor and will not acquire constraint in the form of the axis of rotation, coinciding with its faces. There is no rotation of the cargo relative to the vertical axis, i.e. the cargo is pressed to the floor of the car with previously stretched wires of the same size. Such assumptions suggest that the force of the cargo's impact is evenly distributed along the length of the stop bars, which, in turn, makes it possible to note that the fastener (nail) on it is loaded with evenly distributed loads.

The dynamic model is based on the assumption that cargo securing elements are influenced by spatial force systems, which are perceived by the main constraint (car) and additional constraints (elastic and resistant wooden fasteners). To build a dynamic cargo model (object) with cargo loops in the presence of stop bars, according to the principle of replacing geometric constraints, external constraints are mentally rejected from the object - the platform frame as the main constraint and the elastic fastening elements as additional constraints (see, for example, Fig.2

The influence of the rejected constraints is replaced by reactions $\bar{R}=\left\{\bar{N}, \bar{F}_{\tau}\right\}$ (platform frame) and $\bar{R}_{i}, \bar{R}_{a i}$ (elastic elements of fastenings in one direction, and another direction $\left.-\bar{R}_{p i}, \bar{R}_{a p i}\right) ; R_{\text {bar }}=\left\{R_{\text {bar. } x}, R_{\text {bar. } y}\right\}$ - so far unknown reactions of stop bars, which keep the cargo from shearing both along and across the car. Here, $R_{\text {bar. } y}$ is the unknown resultant reaction of two bars, 5 and 6 (see Fig. 1), laid close to the sides of the cargo. 
The reaction $\bar{R}$ of external constraint is decomposed into normal $\bar{N}$ and tangential $\bar{F}_{\tau}$ components, i.e. $\bar{R}=\bar{N}+\bar{F}_{\tau}$. The tangent component $\bar{F}_{\tau}$ directed along the surface of the car's floor is known to be called the friction force $\bar{F}_{\tau}$ determined by Coulomb's law. The coordinates $x_{R}, y_{R}$ and/or $x_{N}, y_{N}$ - points of application of the reaction of external constraint $\bar{R}=\left\{\bar{N}, \bar{F}_{\tau}\right\}$ are unknown and must be determined.

Active $\left(G, I_{e}, F_{r \mathrm{r}}\right)$ and reactive $\left(R, R_{i}, R_{\mathrm{bar}}\right)$ forces are applied to the object. The active forces are conditionally (formally) applied to the center of mass of the material system (cargo) $C$, but in reality, they are perceived by external constraints and they are directed from the object, reactive forces $\bar{R}=\left\{\bar{N}, \bar{F}_{\tau}\right\}$ - to the object, and the reactive forces of elastic elements of fastenings of one direction $\bar{R}_{i}, \bar{R}_{a i}$ and another direction $\bar{R}_{p i}, \bar{R}_{a p i}$ - from the object.

It should be noted that since the influence of spatial force systems as active forces is experienced by external constraints - a car (main constraint), elastic fastening elements and stop wooden bars (additional constraints), in reality, it is impossible to specify the coordinates of the points of application of these forces. At the same time, in the dynamic model of the object (cargo), these forces are conditionally (mentally) applied to the center of mass of the material system (cargo).

Let us write the equilibrium condition of the cargo in relative motion (rest) in the projections on the coordinate axes, where $F_{i}=\left\{F_{i x}, F_{i y}, F_{i z}\right\}$ - the elastic forces of the elastic cargo securing elements ( $i=1, n_{\mathrm{p}}$ - the number of securing elements, usually chosen depending on the gravity force of the cargo $G) ; F_{r \mathrm{~B}}=\left\{F_{r \mathrm{~B}, x}, F_{r \mathrm{~B}, y}, F_{r \mathrm{~B}, z}\right\}$ aerodynamic drag; $\bar{N}$ and $\bar{F}_{\tau}$ - the normal and tangential components of the reaction of the main constraint (car) $\bar{R} ; R_{\text {bar }}=\left\{R_{\text {bar. } x}, R_{\text {bar. } y}\right\}$-reactions of stop bars.

Let's recall that $F_{i}$ includes the elastic forces of securing elements $R_{i}$ not only from the impact of external forces but also from the force of preliminary wire twists $R 0_{i}$ (usually $20 \mathrm{kN}$ on average [15]), i.e. $F_{i}=\left\{R_{i}, R 0_{i}\right\}$. Therefore, the forces (tension) in the elastic securing elements $\left(R_{i}\right)$ and the reaction of the stop bars $R_{\text {bar. } x}$ and $R_{\text {bar. } y}$ are unknown.

Determination of the cargo shear in the plane of the car's floor. As in [15-16], the cargo shear $\Delta s$ in the plane of the car's floor, $\mathrm{m}$, in the direction of the impact of resultant spatial force system $\Delta F$ is found by (3.86) in [15], and the equivalent stiffness of elastic securing elements in the direction of the impact of spatial force systems $-c_{\text {equiv. }}^{F}$ in projections of securing elements according to (3.86 b) in, $\mathrm{kN} / \mathrm{m}$.

Further, as a special case, the cargo shears are determined both along $\Delta x$ and across $\Delta y$ of the car (m), finding the equivalent stiffness of the securing elements $c_{\text {equiv. } x}$ and $c_{\text {equiv. } y}$ in the direction of impact of the resulting flat force system $\Delta F_{\text {long. }}=\Delta F_{x}$ and $\Delta F_{\text {lat. }}=\Delta F_{y}$. Then the cargo shears can be found both along and across the car, m:

$$
\Delta x=\Delta F_{x} / c_{\text {equiv. } x},
$$




$$
\Delta y=\Delta F_{y} / c_{\text {equiv. } y} .
$$

In practice, the carriage of cargo during shunting collisions at the pitch park of a marshalling station may in fact result in shears of cargo along the car.

Determination of the equivalent stiffness of the elastic cargo securing elements. In terms of the elastic cargo securing element from annealed wire, whose elastic modulus is $\mathrm{E}=$ $1 \cdot 10^{7} \mathrm{kN} / \mathrm{m}^{2}$, and the cross section area is $\mathrm{A}=\mathrm{n}\left(\pi 10^{-6} \mathrm{~d}^{2} / 4\right)(\mathrm{d}$ and $\mathrm{n}$ - the wire diameter (in $\mathrm{mm}$ ) and the number of threads in the wire (in pcs.) of fasteners), formulas for determining the equivalent stiffness of elastic cargo securing elements can be represented through the projections of elastic securing element on the coordinate axis Oxz and Oyz:

$$
\begin{gathered}
c_{\text {equiv. } x}^{F}=7,854 d_{i}^{2} \sum_{i=1}^{n_{\mathrm{p}}} \frac{n_{i}}{l_{i}}\left(f \frac{h_{i}}{l_{i}}+\frac{a_{i}}{l_{i}}\right) \frac{a_{i}}{l_{i}}, \\
c_{\text {equiv . }}^{F}=7,854 d_{i}^{2} \sum_{i=1}^{n_{\mathrm{p}}} \frac{n_{i}}{l_{i}}\left(f \frac{h_{i}}{l_{i}}+\frac{b_{i}}{l_{i}}\right) \frac{b_{i}}{l_{i}} .
\end{gathered}
$$

Determination of the elongation of each elastic securing element. Then the elongation (stretching) of each $i$-th pre-tensioned elastic securing element $\Delta l_{i}$, depending on the cargo shear along (i.e. $\Delta l_{i}^{x}=f(\Delta x)$ ) or across the car (i.e. $\Delta l_{i}^{y}=f(\Delta y)$ ), is similar to, through projections of elastic securing elements on the longitudinal and lateral axis is in the form:

$$
\begin{aligned}
& \Delta l_{i}^{x}=\Delta x \frac{a_{i}}{l_{i}}, \\
& \Delta l_{i}^{y}=\Delta y \frac{b_{i}}{l_{i}} .
\end{aligned}
$$

Here the designations are the same as in (3) and (4).

Thus, elongation in an elastic securing element when exposed to a flat system of forces will occur only when the cargo is sheared in the floor plane along the car by $\Delta x$, and across the car - $\Delta y$. The converse is also true, when $\Delta x=0$ and $\Delta y=0$, the elastic securing elements are not included in the work of keeping the cargo from shearing. In this case, the cargo is held only by fasteners (nail) calculated according to the formulas in technical specifications.

Determination of the tension of each elastic securing element. The tension $R_{i}$ in $i$-th elastic fasteners when exposed to a flat system of forces is determined according to Hooke's law. As applied to the elastic cargo securing element from the annealed wire depending on the diameter $d_{i}$, the number of threads of wire $n_{i}$ should be determined, taking into account (5) and (6) both along and across the car, in the form:

$$
R_{\text {elast. } i}^{x}=7,584 d_{i}^{2} \frac{n_{i}}{l_{i}} \Delta x \frac{a_{i}}{l_{i}} \leq\left[R_{i}\right]
$$




$$
R_{\text {elast. }}^{y}=7,584 d_{i}^{2} \frac{n_{i}}{l_{i}} \Delta y \frac{b_{i}}{l_{i}} \leq\left[R_{i}\right]
$$

Where $\left[R_{\mathrm{i}}\right]$ - the allowable value of the elastic forces in the fastenings, determined by technical specifications, depending on the number of threads $n_{i}$ and the diameter $d_{i}$ of the fastener wire, $\mathrm{kN} ; E A_{i}$ - stiffness of fasteners, as a straight rod working in tension, $\mathrm{kN} ; A_{i}$ - the cross-sectional area of fastener wire $A_{i}=f\left(d_{i}, n_{i}\right), \mathrm{m}^{2}$.

Note that in (7) and (8), it is enough to find $R_{i}$ for a securing element that is short in length, and not for everyone, as in technical specifications.

Thus, the criterion for assessing the loading of the elastic securing elements is their permissible value, which is determined by the Table 20 in technical specifications

Based on the study of the physical model of the mechanical system (object) (see Fig. 1), it is necessary to form a design (or dynamic) model of cargo securing on the car. The design scheme of application of the reaction of inclined stop bars 5 and 6,5 and $5 a$ in the form of $R_{\text {bar } x}$ and $R_{\text {bar. } y}$ to the cargo, both along and across the car, will be taken as shown in Fig. 2 and 3.

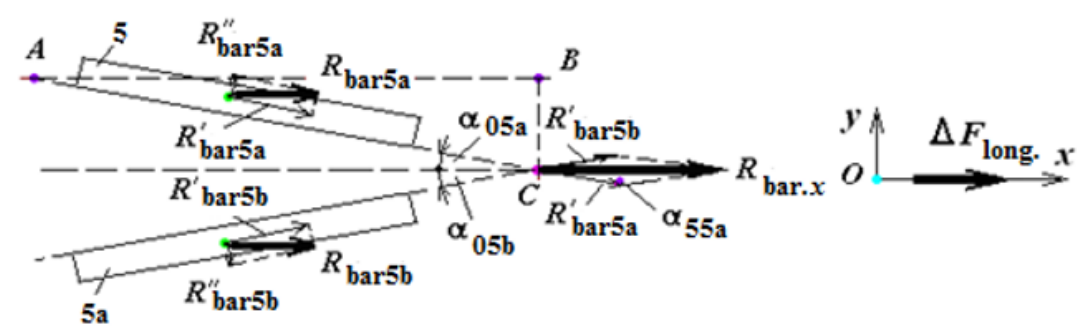

Fig. 2. The scheme of application of the reaction of stop bars 5 and $5 a$ along the car.

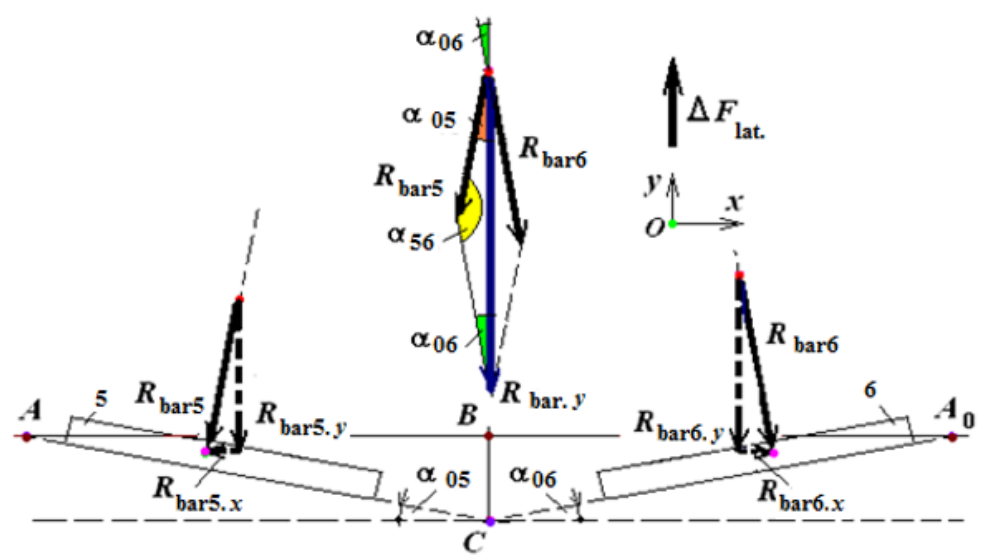

Fig. 3. The scheme of application of the reaction of stop bars 5 and 6 across the car.

Building of mathematical models of cargo securing with inclined side surfaces by means of stop elements (bars) under the influence of spatial force systems. In this case, mathematical models of cargo securing with inclined side surfaces by means of stop elements (bars) will be considered using examples of calculations for keeping cargo from 
shearing separately from the impact of longitudinal forces and separately from lateral forces.

Mathematical models for keeping the load from shearing along the car. We take into account that the shear of cargo along the car will be completely limited only by inclined bars 5 and $5 a$. For example, if the useful area of a carriage allows placing the cargo with specific outlines using stop wooden bars 5 and $5 a$ (see Fig. 2), then the required number of fasteners (nail) needed to fix the stop bars with which the cargo will not shear along the car, can be found by the formula (see (46) in [9]):

$$
n_{\text {nail. } x} \geq \frac{R_{\text {bar. } x}}{n_{\text {bar. } x}\left[R_{\text {nail }}\right]},
$$

where $n_{\text {bar. } x}$ - number of stop and/or spacing bars, pcs. (usually take 1 or 2 pieces); $\left[R_{\text {nail }}\right]$ - permissible load on one fastener (nail), $\mathrm{kN}$ (see Table 32 in [12]). For example, for a nail $\varnothing 4 \mathrm{~mm}$ and a length of $100 \div 120 \mathrm{~mm},\left[R_{\text {nail }}\right]=0.047 \mathrm{kN}$, for $\varnothing 5 \mathrm{~mm}$ and a length of $100 \div 150 \mathrm{~mm},\left[R_{\text {nail }}\right]=0.075 \mathrm{kN}$, and for $\varnothing 6 \mathrm{~mm}$ and a length of $150 \div 200 \mathrm{~mm},\left[R_{\text {nail }}\right]=$ $1.08 \mathrm{kN}$. According to the standard of European countries [8, 18], for fasteners $\varnothing 5 \mathrm{~mm}$ and a length of $100 \div 150 \mathrm{~mm},\left[R_{\text {nail }}\right]=1.25 \mathrm{kN}$, and $\varnothing 6 \mathrm{~mm}$ and a length of $150 \div 200 \mathrm{~mm},\left[R_{\text {nail }}\right]$ $=1.5 \mathrm{kN}$.

In (13) $R_{\text {bar. } x^{-}}$the reaction of stop bars: $R_{\text {bar. } x}=\Delta F_{\text {long. }}$. Here, $\Delta F_{\text {long }}=F_{\text {shear. } x}-F_{\text {ret. } x}$, taking into account the fact that $F_{\text {shear. } x}$ and $F_{\text {ret. } x}$ are "shearing" and "retentive" forces, which will be perceived by stop bars 5 and $5 a$ used as additional means of fastening:

$$
\begin{gathered}
F_{\text {shear } . x}=G_{x}+I_{e x} \\
F_{\text {ret. } x}=F_{r \mathrm{~B} x}+\sum_{i=1}^{n_{\mathrm{p}}} R 0_{x i}+f\left[\left(G_{z}-I_{e z}\right)+F_{r \mathrm{~B} z}+\sum_{i=1}^{n_{\mathrm{p}}} R 0_{z i}\right]
\end{gathered}
$$

where $G_{x}$ and $G_{z}$ - gravity force projections on the $x$ and $z$ axes, $\mathrm{kN}: G_{x}=G \sin \psi_{0}$ and $G_{z}$ $=G \cos \psi_{0} ; \psi_{0}-$ the descent angle of the rolling stock; $i=1, n_{\mathrm{p}}-$ number of fasteners (pieces);

$R 0_{i z}$-projection of the reaction of preliminary twisting of the fastener wire $R 0_{i}$ on the $z$ axis, which is found by the formula:

$$
R 0_{i z}=\sum_{i=1}^{n_{p}} R 0_{i} \frac{h_{i}}{l_{i}}
$$

taking into account the fact that $h_{i}$ - the projection of the $i$-th securing elements of length $l_{i}$ on the vertical, m;

$R 0_{i x}$ - projection of the reaction of preliminary twisting of the fastener wire $R 0_{i}$ on the $x$ axis:

$$
R 0_{i x}=\sum_{i=1}^{n_{\mathrm{p}}} R 0_{i} \frac{a_{i}}{l_{i}}
$$

taking into account the fact that $a_{i}--$ the projection of the $i$-th securing elements of length $l_{i}$ on the horizontal, $\mathrm{m}$. 
In accordance with this, the longitudinal force $\Delta F_{\text {long }}$ perceived by the elastic securing elements, $\mathrm{kN}$, is found by the formula:

$$
\Delta F_{\text {long }}=F_{\text {shear } . x}-F_{\text {ret. } x}
$$

In the limiting case, the equality $F_{\text {shear } . x}=F_{\text {ret. } x}$ holds for $\Delta F_{\text {long. }}=0$. In this case, the cargo will be at rest relative to the floor of the car, i.e. stop bars will still prevent the cargo from shearing along the car.

Shear of cargo along the car will not occur if the following inequality holds:

$$
F_{\text {shear . } x}<F_{\text {ret } . x}
$$

As can be seen, the cargo is also kept from a longitudinal shear by two pairs of stop bars 5 and $5 a$ nailed to the floor of the car close to the two oppositely inclined side surfaces of the cargo $A C$ and $A_{0} C$ (see Fig. 2 and 3). Suppose that according to the drawing data, the geometrical parameters of the inclined side surfaces of the cargo $A B$, $A_{0} B$ and $B C$ are known.

Let's obtain the minimum number of fasteners (nail) $n_{\text {nail. } x}$ required to hold the stop bars 5 and $5 a$ from the impact of the longitudinal force $R_{\text {bar. } x}=\Delta F_{\text {long }}$ (see Fig. 2), conditionally taking the number of stop bars $n_{\text {bar }}=2$ Then, rounding off the calculated value of fasteners $n_{\text {nail. } x}$ upwards, so that it has an even number, we find the reactions of the stop bars using the same formula from technical specifications.

Mathematical solution of the problem in the form of specific analytical formulas for the calculation of inclined stop bars. Note that in the following, it is possible to determine the

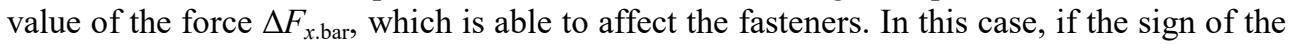
force $\Delta F_{x \text {.bar }}$ turns out to be negative, it means that retaining the cargo from shearing along the car is ensured and elastic securing elements of one direction will not be included in the work of retaining the cargo from shearing in the direction of the impact of longitudinal forces. Conversely, if the sign of the force $\Delta F_{x \text {.bar }}$ turned out to be positive, then this means that the retention of the cargo from shearing along the car is not provided with stop bars 5 and $5 a$, which are inclined towards the side surfaces of the load. In this case, a clear shear of the cargo along the car with the inclusion of elastic securing elements of one direction can occur. In this case, the stop bar 4 nailed to the floor of the car close to the end surface of the cargo (see Fig. 1) will be included in the work to retain the cargo from shearing along the car.

Considering that the angle of inclination of the side surfaces of the stop bars 5 and $5 a$ is the same, i.e. $\alpha_{05 \mathrm{a}}=\alpha_{05 \mathrm{~b}}$ (see Fig. 2), by the cosine theorem, we find the unknown components $R_{\mathrm{bar} 5 \mathrm{a}}^{\prime}=R_{\mathrm{bar} 5 \mathrm{~b}}^{\prime}$ of the longitudinal $R_{\mathrm{bar} . x}=\Delta F_{\text {long. }}$ along the fibers of the stop bar per each stop bar 5 and $5 a$ (see Fig. 3), with the exception of the forces perceived by the stop bar 4 (see Fig. 1):

$$
R_{\text {bars }}^{\prime}=\frac{R_{\text {bar. } x}}{\sqrt{2\left(1-\cos \left(\alpha_{5,5 a}\right)\right)}},
$$

where $\alpha_{5,5 \mathrm{a}}-$ the angle between the reactions of the stop bars 5 and $5 a: \alpha_{5,5 \mathrm{a}}=\pi-\left(\alpha_{05 \mathrm{a}}+\right.$ $\left.\alpha_{056}\right)$.

The angles of inclination of the side surfaces of the stop bars 5 and $5 a$ in the form of $\alpha_{05 \mathrm{a}}$ and $\alpha_{05 \mathrm{~b}}$ are calculated from the geometry of the side surface of the cargo (see Fig. 3): 


$$
\alpha_{05 a}=\operatorname{arctg}\left(\frac{B C}{A B}\right) ; \alpha_{06}=\operatorname{arctg}\left(\frac{B C}{A_{0} B}\right),
$$

where $B C, A B$ and $A_{0} B$-parameters of inclined surface of the cargo (see Fig. 2 or 3 ).

Define the proportion of the longitudinal force $\Delta F_{\text {long. }}=R_{\text {bar. } x}$ per bar 5 in the form (see Fig. 2):

$$
R_{\mathrm{bara}}=\frac{R_{\mathrm{bar} 5 \mathrm{a}}^{\prime}}{\cos \left(\alpha_{05 a}\right)},
$$

considering that each bar is evenly loaded, i.e. $R_{\mathrm{bar} 5 \mathrm{~b}}=R_{\mathrm{bar} 5 \mathrm{a}}$, because $\alpha_{05 \mathrm{~b}}=\alpha_{05 \mathrm{a}}$.

Let's find the component $R_{\mathrm{bar} 5 \mathrm{a}}^{\prime \prime}$ of the force $R_{\mathrm{bar} 5 \mathrm{a}}$ expanding the stop bar 5 across its fibers

$$
R_{\mathrm{bar} 5 \mathrm{a}}^{\prime \prime}=R_{\mathrm{bar} 5 \mathrm{a}} \sin \alpha_{05 a}
$$

Note that the component $R_{\mathrm{bar} 5 \mathrm{a}}^{\prime}$ of the full reaction $R_{\mathrm{bar} 5 \mathrm{a}}$ of the bar 5 , determined by (20) depending on the reaction of the bar along the fibers $R_{\text {bar. } x}=\Delta F_{\text {long. }}$, tends to shear the stop bar along its fiber, and the component $R_{\text {bar5a }}^{\prime \prime}$ - across the fiber. Similarly, the component $R_{\mathrm{bar} 5 \mathrm{~b}}^{\prime}$ of the full reaction $R_{\mathrm{bar} 5 \sigma}$ of the bar $5 b$, also determined by (20) depending on the reaction of the bar along the fibers $R_{\text {bar. } x}=\Delta F_{\text {long., }}$ tends to shear the stop bar along its fiber, and the component $R_{\text {bar5b }}^{\prime \prime}$ - across the fiber.

Let's obtain the minimum number of fasteners (nail) $n_{\text {nail5 }}$ and $n_{\text {nailsa }}$, which are required to retain the stop bars 5 and $5 a$ from the impact of the longitudinal force $\Delta F_{\text {long. }}$ $=R_{\text {bar. } x}$, using the formula from technical specifications $[9,12]$.

We obtain the total number of fasteners $n_{\text {nail }}$ (where $n_{\text {nail }}=n_{\text {nail5 }}+n_{\text {nail5a }}$ ), rounding off the result upwards, so that it has an even number.

It should be borne in mind that if the condition $R_{\mathrm{bar} 5 \mathrm{a}}^{\prime \prime}>>R_{\mathrm{bar} 5 \mathrm{~b}}^{\prime \prime}$ is met, then the action of the force $R_{\mathrm{bar} 5 \mathrm{a}}^{\prime \prime}$ across the fiber does not exclude the probability of splitting the stop bar $5 a$ [15].

In the particular case, if the side surfaces of the cargo are parallel to the longitudinal axis, which corresponds to $\alpha_{05 \mathrm{a}}=\alpha_{05 \mathrm{~b}}=0$, then it will become apparent from formula (16) that the stop bars 5 and $5 a$ are not loaded with the longitudinal force $\Delta F_{\text {long. }}=R_{\text {bar. } x}$ (see Fig. 2). It should be noted that if the shape of the cargo is such that it is not possible to nail the stop bars 5 and $5 a$ (also 6 and $6 a$ ) to the floor of the car using the total number of fasteners (nail) $n_{\text {nail }}$, then it is possible to take $n_{\text {nail }}$ with a smaller amount $n_{\text {naillo }}$, i.e. $n_{\text {nailo }}$ $<n_{\text {nail }}$, and calculate the new value of the reaction $R_{\text {bar0 }}$ from (13) (see (46) in [12]), perceived by the stop bars 5 and $5 a$. Then it is necessary to find the value of the reaction $R_{\text {bar4.x }}$, which will be perceived by stop and/or spacing bars 4 (see Fig. 1), in the form:

$$
R_{\text {bar } 4 . \mathrm{x}}=R_{\text {bar. } x}-R_{\text {bar } 0}
$$

where $R_{\text {bar } 0}=n_{\text {bar } 5} n_{\text {nail0 }}\left[R_{\text {nail }}\right]$, taking into account that $n_{\text {bar } 5}-$ number of the stop bars 5 and $5 a\left(2 \mathrm{pcs}\right.$ ); $\left[R_{\text {nail }}\right]$ - permissible load on one fastener (nail) (see. Table 32 in [12]), $\mathrm{kN}$.

In the particular case, if the configuration of the cargo is such that it allows the use of only stop and/or spacing bars nailed to the floor of the car close to the end surface of the cargo (see Fig. 2 and 3), then the number of fasteners is determined as in [9, 12]. In this 
case, the reaction of the stop bars, according to the action and reaction law of mechanics [19], are equal and opposite in the direction to the longitudinal force $R_{\text {bar. } x}=\left|\Delta F_{\text {long }}\right|$. Then, as in (9) (see (46) in [12]), by the value of $R_{\text {bar. } x}$, by the number of stop and/or spacing bars $n_{\text {bar }}$ and the permissible load on one fastener (nail) $\left[R_{\text {nail }}\right]$, it is possible to find the number of fasteners (nail) $n_{\text {nail }}$, pcs.

Mathematical models for retaining the cargo from shearing across the car. An example of cargo retention across the car in the practice of cargo transportation is presented in Fig. 2 and 3, a method of placing and securing cargo on the car, where the cargo is retained from lateral shear by two pairs of stop bars 5 and 6 nailed to the floor of the car against two oppositely inclined side surfaces of the cargo $A C$ and $C A_{0}$ (see Fig. 3).

The required number of fasteners (nail) needed for fastening the stop bars 5 and 6 , with which the cargo will not shear across the car, can be determined by (46) in [12]:

$$
n_{\text {nail. } y} \geq \frac{R_{\text {bar. } y}}{n_{\text {bar. } y}\left[R_{\text {nail }}\right]},
$$

where $n_{\text {bar. } y}$ - the number of stop and/or spacing bars, pcs. (usually take 1 or 2 pieces); $\left[R_{\text {nail }}\right]$ - the allowable load on one fastener (nail), $\mathrm{kN}$ (see Table 32 in [12]).

In formula (21), $R_{\text {bar. } y^{-}}$the reaction of stop bars: $R_{\text {bar. } y}=\Delta F_{\text {lat. }}$. Here, $R_{\text {bar. } y}=F_{\text {shear. } y}-F_{\text {ret. } y}$, taking into account the fact that $F_{\text {shear. } y}$ and $F_{\text {ret. } y}$ are the "shearing" and "retentive" forces, which will be perceived by a stop bar placed across the car and used as an additional means of fastening (see formulas (15) and (16) in [18]):

$$
\begin{gathered}
F_{\text {shear. } y}=I_{e y}+\left(I_{n}+F_{r \mathrm{~B} . y}\right) \cos \theta \\
F_{\text {ret. } y}=G_{y}+\sum_{i=1}^{n_{\mathrm{p}}} R 0_{y i}+f\left[\left(G_{z}-I_{e z}\right)+\left(I_{n z}+F_{r \mathrm{~B} . z}\right)+\sum_{i=1}^{n_{\mathrm{p}}} R 0_{z i}\right]
\end{gathered}
$$

where $G_{y}$ and $G_{z}$ - projections of gravity on the axes $O y$ and $O z, \mathrm{kN}$ (see (15));

$I_{n z}$ and $F_{r \mathrm{~B}, z}-$ projections of force $I_{n}$ and $F_{r \mathrm{~B} \text {. }}$ on the axis $O z, \mathrm{kN}: I_{n z}=I_{n} \sin \theta$ and $F_{r_{\mathrm{B} . z}}=$ $F_{r \mathrm{~B}} \sin \theta ; i=1, n_{\mathrm{p}}-$ number of fasteners (pcs.);

$R 0_{i z}$ - projections of the reaction of preliminary twisting of fasteners wire $R 0_{i}$ on the axis $O z$, which are found by (16);

$R 0_{i y}$ - projections of the reaction of preliminary twisting of fasteners wire $R 0_{i}$ on the axis $O y$ :

$$
R 0_{i y}=\sum_{i=1}^{n_{p}} R 0_{i} \frac{b_{i}}{l_{i}}
$$

where $b_{i}$ - projection of the $i$-th fastener elements with the length $l_{i}$ on the axis $O y$, $\mathrm{m}$

In accordance with this, the lateral force $\Delta F_{\text {lat. }}$ perceived by elastic securing elements, $\mathrm{kN}$, is found by the formula (see formula (14) in [14]):

$$
\Delta F_{\text {lat. }}=F_{\text {shear. } y}-F_{\text {ret. } y}
$$


In the limiting case, the equality $F_{\text {shear. } y}=F_{\text {ret. } y}$ holds for $\Delta F_{\text {lat. }}=0$. In this case, the cargo will be at rest relative to the floor of the car, i.e. the stop bar will still prevent the cargo from moving across the car.

Shear of cargo across the car will not occur if the following inequality holds

$$
F_{\text {shear. } y}<F_{\text {ret. } y}
$$

In addition, applying the bar reaction $R_{\text {bar.y }}$ to the cargo, according to the action and reaction law of mechanics [19], we write down $R_{\text {bar. } y}=\left|\Delta F_{\text {lat. }}\right|$ (see Fig. 3).

Let us carry out the mathematical solution of the problem in the form of specific analytical formulas for calculating inclined stop bars. We will show the result of decomposition of the resulting reaction $R_{\text {bar. } y}$ of two inclined bars 5 and 6 (see Fig. 2 and 3) laid close to the inclined side surfaces of the cargo $A C$ and $C A_{0}$. According to the cosine theorem, we write down [16]:

$$
R_{\mathrm{bar} . y}=\sqrt{R_{\mathrm{bar} 5}^{2}+R_{\mathrm{bar} 6}^{2}-2 R_{\mathrm{bar} 5} R_{\mathrm{bar} 6} \cos \alpha_{5,6}},
$$

where $\alpha_{5,6}-$ the angle between the reactions of the stop bars 5 and $6: \alpha_{5,6}=\pi-\left(\alpha_{05 \mathrm{a}}+\right.$ $\alpha_{06}$ ), taking into account that $\alpha_{05 a}$ and $\alpha_{06}$ are calculated according to the geometry of the side surface of the cargo (see Fig. 3).

After the reaction $R_{\mathrm{bar} . y}$ is found, its components $R_{\mathrm{bar} 5}$ and $R_{\mathrm{bar} 6}$ can be determined using the sinus theorem [20]

$$
\frac{R_{\text {bar6 }}}{\sin \alpha_{05 a}}=\frac{R_{\text {bar }}}{\sin \alpha_{06}}
$$

from where it follows that

$$
R_{\mathrm{bar} 6}=R_{\mathrm{bar} 5} \frac{\sin \alpha_{05 a}}{\sin \alpha_{06}}
$$

In the particular case, if the side surfaces of the cargo are parallel to the longitudinal axis, which is the case when $\alpha_{05 \mathrm{a}}=\alpha_{06}=0$, then equality $R_{\text {bar. } y}=R_{\text {bar } 5}+R_{\text {bar } 6}$ becomes obvious from the formula (27).

Using the formula (27) and the relation (28), after the elementary mathematical calculations, we can find the reactions $R_{\text {bar } 5}$ of the stop bar 5, in contrast to [16], in the form:

$$
R_{\mathrm{bar} 5}=\frac{R_{\mathrm{bar} . y}}{\sqrt{1+\left(\frac{\sin \alpha_{05 \mathrm{a}}}{\sin \alpha_{06}}\right)^{2}-2 \frac{\sin \alpha_{05 \mathrm{a}}}{\sin \alpha_{06}} \cos \alpha_{5,6}}},
$$

and the reaction $R_{\mathrm{bar} 6}$ of the stop bar 6 is found by (28).

According to the obtained data, one can find the projections of the reaction of the stop bar 5 along and across the car (along the axis $O x$ and $O y$ ) (see Fig. 3):

$$
R_{\mathrm{bar} 5 x}=-R_{\mathrm{bar} 5} \sin \alpha_{05 \mathrm{a}}
$$




$$
R_{\mathrm{bar} 5 y}=-R_{\mathrm{bar} 5} \cos \alpha_{05 \mathrm{a}}
$$

In this case, the reaction $R_{\mathrm{bar} .5 x}$ should be included in the number of "retentive" forces acting on the cargo along the car.

Similarly, we find the projections of the reaction of the stop bar 6 (see Fig. 3) on the cargo both along and across the car, $\mathrm{kN}$ :

$$
\begin{gathered}
R_{\mathrm{bar} 6 x}=R_{\mathrm{bar} 6} \sin \alpha_{06} \\
R_{\mathrm{bar} 6 y}=-R_{\mathrm{bar} 6} \cos \alpha_{06}
\end{gathered}
$$

In this case, the reaction $R_{\text {bar. } 6 x}$ should be included in the number of "shearing" forces acting on the cargo along the car (see formula (14)).

Next, taking the number of stop bars 5 and 6 , for example, $n_{\text {bar. } 5}=1$ and $n_{\text {bar. } 6}=1$, we find the minimum number of fasteners $n_{\text {nail. } 5}$ and $n_{\text {nail. } 6}$ necessary for fastening two stop bars 5 and 6 placed across the car. Then it is possible to determine the value of the force $\Delta F_{\text {bar. } y}$ capable of acting on the fasteners. At the same time, if the sign of the force $\Delta F_{\text {bar.y } y}$ turns out to be negative, it means that retaining the cargo from shearing across the car is provided, and elastic securing elements will not be included in the work of retaining the cargo from shearing in the direction of the lateral forces. And vice versa, if the sign of force $\Delta F_{\text {bar. } y}$ turned out to be positive, it means that the cargo retention across the car is not provided with inclined stop bars 5 and 6 . At the same time, there will be a shear of the cargo across the car with the attachment of elastic securing elements of one direction.

Let's make a specific example of the calculation of the forces acting on the stop cargo securing elements when separately exposed to longitudinal and lateral forces.

1. An example of calculating the reaction of inclined stop bars using the derived analytical formulas (16) - (20). Initial data corresponding to Fig. 1 and 2:

$\Delta F_{\text {long1 }}=616$ - the longitudinal force perceived by the elastic securing elements, $\mathrm{kN}$ (adopted from data previously calculated by (14) for the gravity force of the cargo of 560 $\mathrm{kN})$;

$\Delta F_{\text {long. }}=R_{\text {bar } x}=430$ - the longitudinal force perceived by elastic securing elements, taking into account the projections of the forces of preliminary wire twists $\left(F_{\text {long }} R 0_{\text {elast }}=\right.$ $106.7 \mathrm{kN})$ and the friction force from them $\left(F_{\text {fr.long }} R 0=80 \mathrm{kN}\right)$ (according to previously calculated data), $\mathrm{kN}$;

$n_{\text {bar5 }}=n_{\text {bar } 5 \mathrm{a}}+n_{\text {bar5b }}=2$ - the number of stop bars 5 and $5 a$ (under the condition $n_{\text {bar5a }}=$ $\left.n_{\text {bar5b }}\right)$, pcs.;

$\left[R_{\text {nail }}\right]=1.08$ - the allowable value of force per one fastener $\varnothing 6 \mathrm{~mm}$ and a length of $150 \div 200 \mathrm{~mm}$ according to the Table 22 in technical specifications [12], $\mathrm{kN}$.

Calculation results:

$n_{\text {nail } 5}=R_{\text {bar. } x} /\left(n_{\text {bar5 } 5}\left[R_{\text {nail }}\right]\right)=199$ - the estimated number of stop bars 5 and $5 a$, pcs. (see formula (37) in [12]);

$n_{\text {nail5long }}=200$ - the accepted even number of fasteners for fastening the stop bars 5 and $5 a$, pcs.;

$R_{\text {bar. } x 1}=n_{\text {nail5long }} n_{\text {bar5 }}\left[R_{\text {nail }}\right]=432$ - reactions of the stop bars 5 and $5 a, \mathrm{kN}$.

Then the force capable of acting on the means of cargo securing (elastic and stop means of securing), taking into account the reactions $R_{\text {bar. } x 1}$, turned out to be $\Delta F_{\text {bar. } x}=\Delta F_{\text {long. }}-$ $R_{\text {bar. } x 1}=430-435=-2 \mathrm{kN}$

Note, as before, since the sign of force $\Delta F_{\text {bar. } x}$ turned out to be negative, the retention of cargo from shearing along the car is provided, and the elastic elements of securing of one direction will not be included in the work of retaining the cargo from shearing in the direction of the longitudinal forces. 
Let us consider the case when the configuration of the cargo is such that it does not allow fastening the stop bars 5 and $5 a$ with the accepted number of fasteners (nail) $n_{\text {nailslong, and the }}$ number of fasteners nailed to the stop bars $n_{\text {nail5long0 }}$ is less than previously accepted, i.e. $n_{\text {nail5long } 0}<n_{\text {nail5long. }}$. For example, let $n_{\text {nail5long } 0}=150$ pcs. Then the newly calculated values of the reactions of the stop bars 5 and $5 a R_{\text {bar. } x 0}=n_{\text {nail } 5 \text { long } 0} n_{\text {bar5 } 5}\left[R_{\text {nail }}\right]=324 \mathrm{kN}$.

In this case, the force capable of affecting the means of cargo securing, taking into account the reactions $R_{\text {bar. } x 0}$, turned out to be $\Delta F_{\text {bar. } x}=106 \mathrm{kN}$.

In this case, since the sign of the force $\Delta F_{\text {bar. } x}$ turned out to be positive, the retention of the cargo along the car is not provided by the stop bars 5 and $5 a$, which are inclined to the side surfaces of the cargo. In this case, a clear shear of the cargo along the car can occur with the inclusion of elastic elements of securing of one direction and the stop bar 4 nailed to the floor of the car close to the end side of the cargo into work (see Fig. 1).

In this regard, we will continue the calculation of fasteners for the stop bars 5 and $5 a$.

The angles of inclination of the inclined stop bars 5 and $5 a$, calculated by the formula (21) according to the geometry of the inclined surfaces of these bars, turned out to be $\alpha_{05 \mathrm{a}}=\alpha_{05 \mathrm{~b}}=$ $0.76 \mathrm{rad}$. (or 4.35 degrees). The value of the angle $\alpha_{055 \mathrm{a}}=\pi-\left(\alpha_{05 \mathrm{a}}+\alpha_{05 \sigma}\right)=2.99 \mathrm{rad}$. (171.3 degrees).

The value of the unknown components $R_{\mathrm{bar} 5 \mathrm{a}}^{\prime}=R_{\mathrm{bar} 5 \mathrm{~b}}^{\prime}$ of the longitudinal force $R_{\mathrm{bar} . x}=$ $\Delta F_{\text {long. }}$ along the fibers of the stop bar, calculated by the formula (18), were equal to $R_{\mathrm{bar} 5 \mathrm{a}}^{\prime}=R_{\mathrm{bar} 5 \mathrm{~b}}^{\prime}=53.15 \mathrm{kN}$. The reactions of the stop bars 5 and $5 a R_{\mathrm{bar} 5 \mathrm{a}}=R_{\mathrm{bar} 5 \mathrm{~b}}$ from the longitudinal force $R_{\mathrm{bar} 5 \mathrm{a}}^{\prime}=R_{\mathrm{bar} 5 \mathrm{~b}}^{\prime}$, calculated by (22), turned out to be equal to $R_{\mathrm{bar} 5 \mathrm{a}}=R_{\mathrm{bar} 5 \mathrm{~b}}=$ $53.3 \mathrm{kN}$.

The calculated value of the component $R_{\mathrm{bar} 5 \mathrm{a}}^{\prime \prime}$ of the force $R_{\mathrm{bar} 5 \mathrm{a}}$ expanding the stop bar 5 across its fibers, calculated by (19), turned out to be $R_{\mathrm{bar} 5 \mathrm{a}}^{\prime \prime}=4 \mathrm{kN}$.

Let us consider another possible case where the configuration of the cargo is such that it does not allow fastening the stop bars 5 and $5 a$ with the accepted number of fasteners (nail) $n_{\text {nailslong } 0}$ and the number of fasteners nailed to the stop bars $n_{\text {nail5long01 }}$ is less than previously accepted, i.e. $n_{\text {nail5long01 }}<n_{\text {nail5long0. }}$. For example, let $n_{\text {nail5long } 01}=100$ pcs. Then the newly calculated values of the reactions of the stop bars 5 and $5 a R_{\text {bar } x 01}=n_{\text {nail5long } 0} n_{\text {bar } 5}\left[R_{\text {nail }}\right]=216 \mathrm{kN}$.

In this case, the force capable of affecting the means of cargo securing, taking into account the reactions $R_{\text {bar. } x 01}, \mathrm{kN}$, turned out to be $\Delta F_{\text {bar } 4 . x}=R_{\text {bar } 5 \mathrm{a}}-R_{\text {bar. } x 01}=53.3-216=-162.7 \mathrm{kN}$.

In this case, since the sign of force $\Delta F_{\text {bar } 4 . x}$ turned out to be negative, the retention of cargo along the car is provided by stop bars 5 and $5 a$, which are inclined to the side surfaces of the cargo. In addition, there will not be a clear shear of the cargo along the car with the inclusion of elastic elements of securing of one direction in the work. In this case, the stop bar 4 nailed to the floor of the car close to the end surface of the cargo (see Fig. 1) will not be included in the work to retain the cargo along the car.

2. An example of calculating the reaction of inclined stop bars using the derived analytical formulas (27) - (33). Initial data corresponding to Fig. 1 - 3:

$\Delta F_{\text {lat } 1}=181.5$ - the lateral force perceived by elastic securing elements, $\mathrm{kN}$ (adopted from data previously calculated by (25) for the gravity force of the cargo of $560 \mathrm{kN}$ );

$\Delta F_{\text {lat }}=R_{\text {bar. } y}=66$ - the lateral force perceived by the elastic securing elements, taking into account the projections of the forces of the preliminary wire twists $\left(F_{\text {lat }} R 0_{\text {elast }}=36.1\right.$ $\mathrm{kN})$ and the friction force from them $\left(F_{\text {fr.lat }} R 0=79.4 \mathrm{kN}\right)$ (taken from previously calculated data), $\mathrm{kN}$;

$n_{\text {bar. } y}=n_{\text {bar5a }}+n_{\text {bar6 }}=2$ - the number of stop bars 5 and 6 (under the conditions nbar5a $=$ nbar6), units;

$\left[R_{\text {nail }}\right]=1.08$ - the allowable force value per one fastener $\varnothing 6 \mathrm{~mm}$ and a length of $150 \div$ $200 \mathrm{~mm}$ according to the Table 22 in technical specifications, $\mathrm{kN}$. 
Calculation results:

$n_{\text {nail. } y}=R_{\text {bar. } y} /\left(n_{\text {bar. } y}\left[R_{\text {nail }}\right]\right)=30.6$ - the estimated number of stop bars 5 and 6 , pcs. (see formula (38) in [9]);

$n_{\text {nail.ylat }}=36$ - the accepted even number of fasteners for fastening the stop bars 5 and 6 , pcs .;

$R_{\text {bar. } y 1}=n_{\text {nail.ylat }} n_{\text {bar. } y}\left[R_{\text {nail }}\right]=77.8$ - reactions of the stop bars 5 and $6, \mathrm{kN}$.

Then the force capable of acting on the means of cargo securing (elastic and stop means of securing), taking into account the reactions $R_{\text {bar. } y 1}$, turned out to $\Delta F_{\text {bar. } y 1}=\Delta F_{\text {lat. }}-R_{\text {bar. } y 1}=$ $66-77.8=-11.8 \mathrm{kN}$.

As you can see, the sign of the force $\Delta F_{\text {bar. } y 1}$ is negative. In this case, the retention of the cargo from the shear across the car by the stop bars 5 and 6 is provided. In addition, elastic elements of securing of one direction will not be included in the work on retaining the cargo from shearing in the direction of the impact of lateral forces.

Let us consider the case when the configuration of the cargo is such that it does not allow fastening the stop bars 5 and 6 with the accepted number of fasteners (nail) $n_{\text {nail.ylat }}$ and the number of fasteners nailed to the stop bars $n_{\text {nail.ylat } 0}$ is less than previously accepted, i.e. $n_{\text {nail.ylat } 0}$ $<n_{\text {nail.ylat }}$. For example, let $n_{\text {nail.ylat } 0}=20$ pcs. Then the newly calculated values of the reactions of the stop bars 5 and $6 R_{\text {bar. } y 01}=n_{\text {nail.ylat } 0} n_{\text {bar } y}\left[R_{\text {nail }}\right]=43.2 \mathrm{kN}$.

In this case, the force capable of acting on the means of cargo securing, taking into account the reactions $R_{\text {bar. } y 01}$, turned out to be $\Delta F_{\text {bar. } y 1}=22.8 \mathrm{kN}$.

Then, since the sign of force $\Delta F_{\text {bar. } y 1}$ turned out to be positive, retention of the cargo across the car is not provided by the stop bars 5 and 6 . In this case, a clear shear of the cargo across the car can occur with the inclusion of elastic elements of securing of one direction in the work (see Fig. 1).

In this regard, we continue the calculation of fasteners for the stop bars 5 and 6 . As before, let the inclination angles of the inclined stop bars 5 and 6 , calculated by (21) according to the geometry of the inclined surfaces of these bars, be $\alpha_{05 \mathrm{a}}=0.76 \mathrm{rad}$. (or 4.35 degrees) and $\alpha_{06}=$ $0.065 \mathrm{rad}$. (or 3.7 degrees). The value of the angle $\alpha_{056}=\pi-\left(\alpha_{05 \mathrm{a}}+\alpha_{6}\right)=3 \mathrm{rad}$. (171.3 degrees).

The reaction values of the stop bars 5 and 6 , calculated by (28) and (29), and directed across their fibers, turned out to be $R_{\mathrm{bar} 5}=10.52$ and $R_{\mathrm{bar} 6}=12.34 \mathrm{kN}$, respectively.

The projections of the reaction of the stop bar 5 along and across the car (along the axis $O x$ and $O y$ ), calculated by (30) and (31), were $R_{\mathrm{bar} 5 x}=-0.8$ and $R_{\mathrm{bar} 5 y}=-10.5 \mathrm{kN}$, respectively. In this case, the reaction $R_{\mathrm{bar} .5 x}$ should be included in the number of "retentive" forces acting on the cargo along the car (see formula (15)).

Similarly, we find the projections of the reaction of the stop bar 6 (see Fig. 3) along and across the car, calculated by (32) and (33), which were $R_{\mathrm{bar} 6 x}=0.8$ and $R_{\mathrm{bar} 6 y}=-$ $12.32 \mathrm{kN}$, respectively. In this case, the reaction $R_{\text {bar.6x }}$ should be included in the number of "shearing" forces acting on the cargo along the car (see formula (14)).

Next, taking the number of stop bars 5 and 6 , for example, $n_{\text {bar } 5}=1$ and $n_{\text {bar6 }}=1$, we find the minimum number of fasteners $n_{\text {nail } 5}$ and $n_{\text {nail }}$ necessary for fastening two stop bars 5 and 6 placed across the car.

Calculation results:

$n_{\text {nail } 5}=R_{\text {bar5 }} /\left(n_{\text {bar5 }}\left[R_{\text {nail }}\right]\right)=10.5 /(1 \times 1.08)=9.74-$ the estimated number of the stop bars 5 , pcs.;

$n_{\text {nail6 }}=R_{\text {bar6 }} /\left(n_{\text {bar6 }}\left[R_{\text {nail }}\right]\right)=12.34 /(1 \times 1.08)=11.43-$ the estimated number of the stop bars 6 , pes.;

$n_{\text {nail.long } 5}=10$ and $n_{\text {nail.long } 6}=12$ - the accepted even numbers of fasteners for fastening the stop bars 5 and 6 , pcs.; 
$n_{\text {nail56 }}=n_{\text {nail.long5 }}+n_{\text {nail.long6 }}=22-$ total number of fasteners for fastening two stop bars 5 and 6 , pcs.;

$n_{\text {bar56 }}=n_{\text {bar5 }}+n_{\text {bar6 }}=2-$ the accepted quantities of two stop bars 5 and 6 , pcs.;

$R_{\text {bar. } y 56}=n_{\text {nail } 56} n_{\text {bar56 }}\left[R_{\text {nail }}\right]=47.2-$ the reaction of the stop bars 5 and $6, \mathrm{kN}$.

Then the force capable of acting on the means of cargo securing (elastic and stop means of securing), taking into account the reactions $R_{6 \mathrm{p} . y 56}$, turned out to be $\Delta F_{\text {bar56 }}=$ $\Delta F_{y 01}-R_{\text {bar } y 56}=22.8-47.2=-24.7 \mathrm{kN}$.

As you can see, the sign of the force $\Delta F_{\text {bar56 }}$ turned out to be negative. This means that there will not be a shear of the cargo across the car, and the elastic elements of securing of the same direction will not be included in the work of retaining the cargo from shearing in the direction of the lateral forces.

Finite analytical formulas for calculating the reaction of inclined stop bars under the influence of a spatial force system. Let's consider finding the reaction of stop bars $5,5 a$ and 6 when exposed to a spatial force system: $\bar{R}_{\text {bar. } x}=\left|\Delta \bar{F}_{\text {long }}\right|$ and $\bar{R}_{\text {bar. } y}=\left|\Delta \bar{F}_{\text {lat }}\right|$ (see Fig. 2 and 3).

The reaction of the stop bar 5 along the car should be found by the formula

$$
R_{\mathrm{bar} 5 \mathrm{a}}^{0}=R_{\mathrm{bar} 5 \mathrm{a}}-R_{\mathrm{bar} 5 \mathrm{x}}
$$

The reaction of the stop bar 5 along the fibers -

$$
R_{\mathrm{bar} 5 \mathrm{a}}^{01}=R_{\mathrm{bar} 5 \mathrm{a}}^{0} \cos \alpha_{05 \mathrm{a}}
$$

and across the fibers -

$$
R_{\text {bar } 5 \mathrm{a}}^{02}=R_{\text {bar } 5}-R_{\text {bar } 5 \mathrm{a}}
$$

The reactions of the stop bar $5 a$ along the car should be found by (22) and (23).

The reaction of the stop bar 6 along the car should be found by the formula

$$
R_{\mathrm{bar} 6}^{0}=R_{\mathrm{bar} 6}+R_{\mathrm{bar} 6 \mathrm{x}}
$$

The reaction of the stop bar 6 along the fibers -

$$
R_{\mathrm{bar} 6}^{01}=R_{\mathrm{bar} 6 \mathrm{x}} \cos \left(\alpha_{06}\right)
$$

or, taking into account (32),

$$
R_{\mathrm{bar} 6}^{01}=R_{\mathrm{bar} 6} \sin \left(\alpha_{06}\right) \cos \left(\alpha_{06}\right)
$$

or, taking into account the formula of double angles [20],

$$
R_{\mathrm{bar} 6}^{01}=\frac{R_{\mathrm{bar} 6}}{2} \sin \left(2 \alpha_{06}\right)
$$

and across the fibers - find by (28).

3. An example of the calculation of inclined stop bars using the derived analytical formulas (34) - (39) and the calculated data in examples 1 and 2, obtained by formulas (16) - (19) and (27) - (33). Calculation results:

$$
\begin{aligned}
& R_{\text {bar } 5 \mathrm{a}}^{0}=R_{\text {bar } 5 \mathrm{a}}-R_{\mathrm{bar} 5 \mathrm{x}}=53.31-(-0.8)=54.1-\text { the reaction of the stop bar } 5 \text { along the car; } \\
& R_{\mathrm{bar} 5 \mathrm{a}}^{01}=R_{\mathrm{bar} 5 \mathrm{a}}^{0} \cos \alpha_{05 \mathrm{a}}=54.1 \times 0.997=53.95-\text { the reaction of the stop bar } 5 \text { along the fiber; }
\end{aligned}
$$


$R_{\mathrm{bar} 5 \mathrm{a}}^{02}=R_{\mathrm{bar} 5}-R_{\mathrm{bar} 5 \mathrm{a}}=10.52-53.31=-42.78-$ the reaction of the stop bar 5 across the fiber;

$R_{\mathrm{bar} 6}^{0}=R_{\mathrm{bar} 6}+R_{\mathrm{bar} 6 \mathrm{x}}=12.34+0.8=13.14-$ the reaction of the stop bar 6 along the car;

$R_{\mathrm{bar} 6}^{01}=R_{\mathrm{bar} 6 \mathrm{x}} \cos \alpha_{06}=0.8 \times 0.998=0.797-$ the reaction of the stop bar 6 along the fiber;

$R_{\text {bar6 }}=12.34-$ the reaction of the stop bar 6 across the fiber, $\mathrm{kN}$.

Thus, the calculation of the number of fasteners (nail) for fastening stop bars to the floor of the car under the influence of both flat and spatial force systems.

\section{Discussion}

Summarizing the results of the study performed, we will especially note the following.

1. The problem's conditions and accepted assumptions are formulated when creating a dynamic model, taking into account the fact that in a dynamic model, cargo securing elements are affected by spatial force systems that are perceived by the main constraint (car) and additional constraints (elastic and stop wooden fasteners).

2. Based on the study of the physical model of the mechanical system (object), a design model of securing the cargo on the car is formed, which allowed the construction of mathematical models of its fasteners with inclined side surfaces by means of stop elements (bars) under the influence of spatial force systems.

3. The mathematical solution to the problem of determining the reaction of inclined stop bars under the influence of a spatial force system is implemented. For example, the final analytical formulas for calculating the reaction of inclined stop bars as one of the cargo securing elements on the railway platform are written in a form convenient for calculating.

4. Using final analytical formulas for calculating the reaction of inclined stop bars, specific examples of calculating the forces acting on the stop elements of the cargo securing were made.

The results of previously published papers (see, for example, [3, 9, 10, 12 - 15]) are particular cases of this study. So, for example, when the angles of inclination of the side surfaces of the stop bars 5 and 6 in the form of $\alpha_{05}$ and $\alpha_{06}$ are zero (ie, $\alpha_{05}=0$ and $\alpha_{06}=0$ ), the reactions of the stop bars 5 and 6 are determined in the same way as in $[3,9,10,12-15]$ when exposed only to the lateral forces.

The results of this study are of theoretical significance, since the final analytical formulas for calculating the reaction of inclined stop elements to the side surfaces of the cargo and the decomposition of the projection of these reactions in the direction of the axes of coordinates are given for the first time. Of practical interest for the carriage of cargo on a railway platform (car) is the determination of the number of fasteners (nail) needed to fasten the stop elements to the floor of the car.

Further studies should be conducted, the results of which allow determining the reaction of stop elements for cargo that rely on the floor of the car with an optional configuration.

\section{References}

1. M. Tumielewiez, Ladowa nie zabezpieczanie przesyiek miedzynarodowych. Eksplontacija Kiei, № 10 Richting verladen in Güterwagen, Deine Bahn, 23 (5), 274277 (1995)

2. R. Munzert, Bebadetechnik and Ldung - Sicherund, Deine Bahn, 26(6) 345-348 (1998)

3. Zurrmittel zum Verzuren von Lastenl Dolerych V, DNF: Int. Fachzeitschr. Forger, Lager - and Transporttechn. DHF: Dtsch/ Hebe - and Forgertechn, 44, №5, pp. 50-60 (1998) 
4. http://www.vinnova.se/upload / EPiStorePDF /vr-04-05.pdf. Last accessed 2018/06/07

5. Tekhnicheskiye usloviya razmeshcheniya $\mathrm{i}$ krepleniya gruzov $\mathrm{v}$ vagonakh $\mathrm{i}$ konteynerakh. Moscow, Yurtrans, p. 544 (in Russian) (2003)

6. http://www.nvfnorden.org/lisalib/getfile.aspx?itemid=1593 Last accessed 2018/06/09

7. http://www.highwaystarmagazine.com/images /Driver_Handbook.pdf. Last accessed 2018/07/09

8. Kh. Turanov, E. Timukhina, Analytical modeling cargoes displacement in wagon and tension in fastening. Transp. Prob., 3, № 3, pp. 69-76 (2008)

9. Kh. Turanov, Analytical basis of asymmetrical allocation of cargo masses common centre in wagons. Transp. Prob.. Poland, 4, № 1, pp. 77-86 (2009)

10. Kh. Turanov, Theoretical mechanics in cargo handling problem. Monograph. Novosibirsk, Nauka, p. 376 (2009)

11. Kh. Turanov, Analytical investigation of cargo motion lengthwise the wagon under the action of plane force system. Global J. of Res. in Eng.: A. Mechanical and Mechanics Eng.. 13(10), Version 1.0 Year. New York, pp. 7-16 (2013)

12. Kh. Turanov, Mathematical modeling of cargo motion crosswise the wagon under the action of plane force system. Scie. and Tech., 5(1), pp. 5-14, -JSSN: 2163-2669 (2015) ID: 104000126 , doi: $10.5923 /$ j.scit.2015 0501.02 